\title{
Distance Desert Automata and the Star Height One Problem ${ }^{\star}$ \\ (Extended Abstract) $)^{\star \star}$
}

\author{
Daniel Kirsten \\ LIAFA, Université Denis Diderot - Case 7014, 2 place Jussieu, \\ F-75251 Paris Cedex 05, France
}

\begin{abstract}
We show that it is decidable in time complexity $2^{2^{2^{\mathcal{O}(n)}}}$ whether the language accepted by an $n$-state non-deterministic automaton is of star height one, which is the first ever complexity result for the star height one problem. To achieve this, we introduce distance desert automata as a joint generalization of distance automata and desert automata, and show the decidability of its limitedness problem by solving the underlying Burnside problem.
\end{abstract}

\section{Introduction}

This paper is the second one in a series of papers in which we will introduce new models of weighted automata to solve important decision problems in the theory of recognizable languages. Our first main result states the first ever complexity bound for the "classic" star height one problem. "Classic" star height concerns rational expressions with union, concatenation, and iteration in contrast to extended star height which also allows intersection and complement. To achieve this, we introduce distance desert automata as a joint generalization of K. HASHIGUCHI's distance automata 3] and the author's desert automata 5 . Our second main result is the decidability of the limitedness problem of distance desert automata which generalizes classic results by K. HAsHiGUCHI, H. LEUNG, I. Simon, and the author 35,916]. We prove the decidability of the limitedness problem by a reduction to a Burnside problem which is solved by a fusion and further development of approaches by I. Simon, H. LEunG, and the author 5 , 9,10,14,16.

\section{Overview}

\subsection{Preliminaries}

For sets $M$, we denote by $\mathcal{P}(M)$ the power set of $M$, and we denote by $\mathcal{P}_{f}(M)$ (resp. $\left.\mathcal{P}_{n e}(M)\right)$ the set of all finite (resp. non-empty) subsets of $M$. In the main

* Supported by the grant KI 822/1-1 of the German Research Community (DFG). On leave from Institute of Algebra, Dresden University of Technology, Germany.

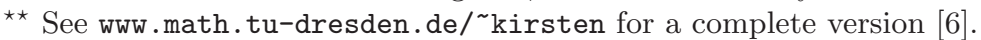


part of the paper, we fix some $n \geq 1$ for the dimension of matrices. Whenever we do not explicitly state the range of a variable, then it ranges over $\{1, \ldots, n\}$, e.g., a phrase like "for every $i, j$ " is understood as "for every $i, j \in\{1, \ldots, n\}$ ".

\subsection{Distance Desert Automata}

A distance desert automaton (dd-automaton) is a 6-tuple $\left[Q, E, I, F, E^{<}, E^{\curlyvee}\right]$ where $[Q, E, I, F]$ is a non-deterministic finite automaton, $E^{<} \subseteq E$ are called péages and $E^{\curlyvee} \subseteq E$ are called water transitions. Let $\mathcal{A}=\left[Q, E, I, F, E^{<}, E^{\curlyvee}\right]$ be a dd-automaton. Its language $L(\mathcal{A})$ is defined as $L([Q, E, I, F])$.

Let $\pi$ be a path in $\mathcal{A}$. We denote by $\Delta_{1}(\pi)$ the number of occurrences of péages in $\pi$. We call $\pi^{\prime}$ a subpath of $\pi$ if there are paths $\pi_{1}, \pi_{2}$ in $\mathcal{A}$ satisfying $\pi=\pi_{1} \pi^{\prime} \pi_{2}$. We denote by $\Delta_{2}(\pi)$ the length of a longest subpath of $\pi$ which does not contain any water transition. The intuition behind these mappings is that we imagine $\pi$ as a path through a desert. We intend to walk along $\pi$. Whenever we came along a péage, we have to pay a coin, i.e., $\Delta_{1}(\pi)$ is the number of coins which are required. We carry a water tank, but this tank does not last the entire path. Whenever we come along a water transition, we can fill up the tank, and the tank has to last until we meet the next water transition. We can understand $\Delta_{2}(\pi)$ as the required capacity of the tank to walk along the path $\pi$.

We define $\Delta(\pi)=\Delta_{1}(\pi)+\Delta_{2}(\pi)$. For every word $w \in \Sigma^{*}$, let us set $\Delta(w)=\min \{\Delta(\pi) \mid p \in I, q \in F, \pi \in p \stackrel{w}{\sim} q\}$, where $p \stackrel{w}{\sim} q$ denotes the set of all paths from $p$ to $q$ with the label $w$. A dd-automaton is limited if there is a $d \in \mathbb{N}$ such that $\Delta(w) \leq d$ for every $w \in L(\mathcal{A})$.

If $E^{\curlyvee}=E$, then $\Delta_{2}(\pi)=0$ for every path $\pi$. In this case, $\mathcal{A}$ is a distance automaton. Consequently, K. HASHiguCHI's distance automata 3 are exactly the dd-automata with $E^{\curlyvee}=E$.

If $E^{<}=\emptyset$, then $\Delta_{1}(\pi)=0$ for every path $\pi$, i.e., $\mathcal{A}$ is a desert automaton. Thus, the author's desert automata $\left[5\right.$ are exactly the dd-automata with $E^{<}=\emptyset$.

A main result of the present paper is the following theorem:

Theorem 1. It is decidable in time complexity $2^{\mathcal{O}\left(n^{2}\right)}$ whether an $n$-state distance desert automaton is limited.

Theorem 1 includes the decidability of the limitedness problems for distance automata 391016] and for desert automata [5].

In Section 3 we reduce the limitedness problem for dd-automata to a Burnside problem. This Burnside problem is a joint generalization of the two Burnside problems corresponding to the limitedness problems of distance (resp. desert) automata [105. It is solved in Section 4 .

The limitedness problem for dd-automata is PSPACE-hard, because it is PSPACE-complete (resp. PSPACE-hard) for distance (resp. desert) automata [1115]. It is undecidable whether two dd-automata define the same mapping, because the same problem is already undecidable for distance automata 8 .

As an application of Theorem 1, we show in Section 5 the following theorem: 
Theorem 2. It is decidable in time complexity $2^{2^{2^{\mathcal{O}(n)}}}$ whether the language of an n-state non-deterministic automaton is of star height one.

The decidability was shown by K. HAshiguCHI in 1982 [4]. However, the complexity which can be estimated from his paper is much larger [12, Annexe B].

\section{An Algebraic Framework for the Limitedness Problem}

We develop an algebraic framework to show the decidability of the limitedness problem for dd-automata. Let $\mathcal{A}=\left[Q, E, I, F, E^{<}, E^{\curlyvee}\right]$ be a dd-automaton, let $n=|Q|$, and assume $Q=\{1, \ldots, n\}$.

\subsection{Finite Semigroup Theory}

We introduce some basic notions from finite semigroup theory. For a deeper understanding, we refer the reader to, e.g., [13]. Let $S$ be a finite semigroup. An element $1 \in S$ is called an identity if $1 p=p 1=p$ for every $p \in S$. If there is no identity in $S$, then we denote by $S^{1}$ the semigroup consisting of the set $S \bullet 1$, on which the operation of $S$ is extended in a way that 1 is the identity of $S^{1}$. If $S$ has an identity, then we define $S^{1}$ to be $S$.

Let $a, b \in S$. If $a \in S^{1} b S^{1}$, or equivalently if $S^{1} a S^{1} \subseteq S^{1} b S^{1}$, then we write $a \leq_{\mathrm{J}} b$. If $a \leq_{\mathrm{J}} b$ and $b \leq_{\mathrm{J}} a$, or equivalently if $S^{1} a S^{1}=S^{1} b S^{1}$, then we write $a=b$. The relation $\Rightarrow$ is an equivalence. Its equivalence classes are called Jclasses. We call some subset $I \subseteq S$ an ideal if $S^{1} I S^{1} \subseteq S$. Every ideal is a union of J-classes.

Let $a, b \in S$. If $a \in S^{1} b$, or equivalently if $S^{1} a \subseteq S^{1} b$, then we write $a \leq_{\mathrm{L}} b$. If $a \leq_{\mathrm{L}} b$, then $a c \leq_{\mathrm{L}} b c$ for every $c \in S$. If $a \leq_{\mathrm{L}} b$ and $b \leq_{\mathrm{L}} a$, or equivalently if $S^{1} a=S^{1} b$, then we write $a=_{\mathrm{L}} b$. We define the relations $\leq_{\mathrm{R}}$ and $=_{\mathrm{R}}$ in the straightforward dual way.

An element $e \in S$ is called an idempotent if $e^{2}=e$. An element $a \in S$ is called regular if there is an idempotent in the J-class of $a$. The sets of idempotents (resp. regular elements) of $S$ are denoted by $\mathrm{E}(S)(\operatorname{resp} . \operatorname{Reg}(S))$.

For every $m \geq 1$, we call $a_{1}, \ldots, a_{m} \in S$ a smooth product if we have $a_{1}=$ $\ldots \Rightarrow a_{m} \Rightarrow\left(a_{1} \ldots a_{m}\right) \in \operatorname{Reg}(S)$.

We call a mapping $\sharp: \mathrm{E}(S) \rightarrow \mathrm{E}(S)$ consistent if we have for every $e, f \in \mathrm{E}(S)$, $a, b \in S$ satisfying $e \Rightarrow f$ and $f=a e b, f^{\sharp}=a e^{\sharp} b$. It is shown in [5] that a mapping is consistent iff we have for every $a, b \in S$ with $a b, b a \in \mathrm{E}(S)(a b)^{\sharp}=$ $a(b a)^{\sharp} b$. It was already observed in [9] that every consistent mapping $\sharp$ admits an extension to $\sharp: \operatorname{Reg}(S) \rightarrow \operatorname{Reg}(S)$ by setting for every $e \in \mathrm{E}(S)$ and $c, d \in S$ satisfying $e=c e d,(c e d)^{\sharp}=c e^{\sharp} d$.

If $a, b \in S$ are a smooth product, then $(a b)^{\sharp}=a^{\sharp} b^{\sharp}=a^{\sharp} b=a b^{\sharp} \quad[5]$.

Let $a \in \operatorname{Reg}(S)$. There are $e, f \in \mathrm{E}(S)$ with $e=_{\mathrm{R}} a=_{\mathrm{L}} f$, i.e., $e a=a=a f$. Thus, $e^{\sharp} a=a^{\sharp}=a f^{\sharp}$, and moreover $a^{\sharp} \leq_{\mathrm{L}} a$ and $a^{\sharp} \leq_{\mathrm{R}} a$. 


\subsection{The Semigroup of Word Matrices}

We extend the notion of word matrices from [5. We consider the alphabet $S=\{\curlyvee, \mathbb{M}, \angle, \not{Z}\}$. The symbol $\curlyvee$ (resp. $\mathbb{M}, \angle$ ) should be pronounced "water" (resp. "desert", "péage"). The words over $S$ represent paths in desert automata. Consider the semiring $\mathbb{S}=\left(\mathcal{P}_{f}\left(S^{+}\right) \cup\{\omega\}, \cup, \cdot\right)$, where $\omega$ is a new element and $\cup$ and . are extended from $\mathcal{P}_{f}\left(S^{+}\right)$by setting for every $X \in \mathbb{S} \backslash\{\emptyset\}$, $\omega \cdot X=X \cdot \omega=\omega, \omega \cup X=X \cup \omega=X$, and further, $\emptyset \cdot \omega=\omega \cdot \emptyset=\emptyset$, $\emptyset \cup \omega=\omega \cup \emptyset=\omega$. The natural ordering on $\mathbb{S}$ is set inclusion extended from $\mathcal{P}_{f}\left(S^{+}\right)$in a way that $\omega$ is between the empty set and the singletons, i.e., we have $\emptyset \subseteq \omega \subseteq X$.

We call the matrices over $\mathbb{S}$ word matrices and denote by $\mathbb{S}_{n \times n}$ the semigroup of $n \times n$-matrices over $\mathbb{S}$. We use the free semigroup $\mathbb{S}_{n \times n}^{+}$over $\mathbb{S}_{n \times n}$. We denote the homomorphism which arises from the identity on letters by $\alpha: \mathbb{S}_{n \times n}^{+} \rightarrow \mathbb{S}_{n \times n}$.

\subsection{On the Semantics of Distance Desert Automata}

We give another method to define the semantics of dd-automata using matrices.

We define a mapping $\theta: E \rightarrow S$, by

$$
\theta(e)= \begin{cases}M & \text { if } e \notin E^{\curlyvee}, e \notin E^{<}, \\ \curlyvee & \text { if } e \in E^{\curlyvee}, e \notin E^{<}, \\ \angle & \text { if } e \notin E^{\curlyvee}, e \in E^{<}, \\ \not & \text { if } e \in E^{\curlyvee}, e \in E^{<} .\end{cases}
$$

This mapping extends to a homomorphism $\theta:\left(\mathcal{P}_{f}\left(E^{+}\right), \cup, \cdot\right) \rightarrow(\mathbb{S}, \cup, \cdot)$. We can assign every $w \in \Sigma^{+}$a matrix $\theta(w) \in \mathbb{S}_{n \times n}$ by setting $\theta(w)[i, j]=\theta(i \stackrel{w}{\sim} j)$. Clearly, $\theta: \Sigma^{+} \rightarrow \mathbb{S}_{n \times n}$ is a homomorphism. The distance function $\Delta$ on paths from Section 2.2 induces a distance function on $S^{+}$as

$$
\Delta(\pi)=|\pi|_{\angle}+|\pi|_{\not \mathcal{Y}}+\max \left\{\left|\pi^{\prime}\right| \mid \pi^{\prime} \in\{\mathbb{M}, \angle\}^{*}, \pi^{\prime} \text { is a subword of } \pi\right\}
$$

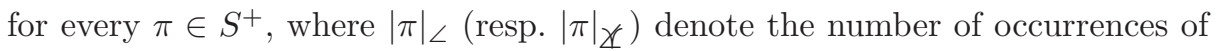
$\angle$ (resp. $\not \Varangle)$ in $\pi$ and $\left|\pi^{\prime}\right|$ denotes the length of $\pi^{\prime}$. We extend $\Delta$ to $X \in \mathbb{S}$ by setting $\Delta(X)=\min \{\Delta(\pi) \mid \pi \in X\}$, if $X \neq \omega$ and $\Delta(\omega)=\omega$.

We can give another definition of the semantics of a desert automaton. For $w \in \Sigma^{+}$, let $\Delta(w)=\min \{\Delta(\theta(w)[i, j]) \mid i \in I, j \in F\}$. This is equivalent to the definition in Section 2.2 up to the empty word.

\subsection{The Distance Desert Semiring}

Let $\mathcal{S}=\{\curlyvee, \mathbb{M}, \angle, \omega, \infty\}$. Intuitively, $\curlyvee$ represents a path with a water transition but without péage, $\mathbb{X}$ represents a path without a water transition and without péage, and $\angle$ represents path with a péage, regardless of whether it contains a water transition. We define on $\mathcal{S}$ an operation - as the maximum for the ordering $M \sqsubseteq \curlyvee \sqsubseteq \angle \sqsubseteq \omega \sqsubseteq \infty$. The operation · corresponds to the concatenation of 
paths, e.g., $\mathbf{M} \cdot \angle=\angle$ means that the concatenation of a path without a water transition and without a péage and a path with a péage yields a path with a péage. Clearly, $(\mathcal{S}, \cdot)$ is a monoid with identity $\mathbb{M}$ and zero $\infty$.

We define $\min$ on $\mathcal{S}$ for the ordering $\curlyvee \leq \mathbb{X} \leq L \leq \omega \leq \infty$. The relation $\leq$ represents something like choice, e.g., $\curlyvee \leq \mathbb{M}$ means that we rather choose a path with a water transition but without a péage than a path without water transition and without péage. The operation · is stable w.r.t. $\leq$. Moreover, $(\mathcal{S}, \min , \cdot)$ is a semiring with zero $\infty$ and identity $\mathbf{X}$.

Let $\Psi: S \rightarrow \mathcal{S}$ be the mapping defined by $\Psi(\curlyvee)=\curlyvee, \Psi(\mathbb{X})=\mathbb{X}$, and $\Psi(\angle)=\Psi(\not{X})=\angle$. It extends to homomorphisms $\Psi:\left(S^{+}, \cdot\right) \rightarrow(\mathcal{S}, \cdot)$ and $\Psi:(\mathbb{S}, \cup, \cdot) \rightarrow(\mathcal{S}, \min , \cdot)$ where $\Psi(\emptyset)=\infty$ and $\Psi(\omega)=\omega$. For every $X \in \mathbb{S}$,

$$
\Psi(X)= \begin{cases}\curlyvee & \text { if } X \text { contains a word } \pi \in\{\curlyvee, \mathbb{A}\}^{*} \curlyvee\{\curlyvee, \mathbb{A}\}^{*} \\ \mathbb{X} & \text { if } X \text { contains a word } \pi \in \mathbb{A}^{+}, \text {but } X \cap\{\curlyvee, \mathbb{A}\}^{*} \curlyvee\{\curlyvee, \mathbb{A}\}^{*}=\emptyset \\ \angle & \text { if } X \text { contains a word } \pi \in S^{*}\{\angle, \not Y\} S^{*}, \text { but } X \cap\{\curlyvee, \mathbb{A}\}^{+}=\emptyset \\ \omega & \text { if } X=\omega \\ \infty & \text { if } X=\emptyset .\end{cases}
$$

Clearly, $\Psi$ extends to a homomorphism $\Psi: \mathbb{S}_{n \times n} \rightarrow \mathcal{S}_{n \times n}$.

For technical reasons, we need a more specific distance function on $\mathbb{S}$. For every $X \in \mathbb{S}$ and $z \in\{\curlyvee, \mathbb{M}, \angle\}$, let $\Delta(X, z)=\min \{\Delta(\pi) \mid \pi \in X, \Psi(\pi)=z\}$. For every $z \in\{\curlyvee, \mathbb{X} \backslash, \angle\}$, we have $\Delta(\omega, z)=\Delta(\emptyset, z)=\infty$. For $X \in \mathbb{S} \backslash\{\emptyset\}$, let

$$
\Delta^{\prime}(X)= \begin{cases}\Delta(X, \Psi(X)) & \text { if } X \in \mathbb{S} \backslash\{\omega\} \\ \omega & \text { if } X=\omega\end{cases}
$$

For every $X \in \mathbb{S} \backslash\{\omega, \emptyset\}$, we have $\Delta(X)=\min \{\Delta(X, z) \mid z \in\{\curlyvee, \mathbb{M}, \angle\}\} \leq \Delta^{\prime}(X)$.

\subsection{Strange Limits}

We generalize the notion of a $\Psi$-limit from [5]. A $\Psi$-limit of some sequence over $\mathbb{S}$ describes in terms of $\mathcal{S}$ how the sequence is bounded.

Recall that some sequence $\left(q_{k}\right)_{k \geq 1}$ is a subsequence of $\left(p_{k}\right)_{k \geq 1}$ if there is a strictly increasing mapping $f: \mathbb{N} \rightarrow \mathbb{N}$ such that $q_{k}=p_{f(k)}$ for every $k \geq 1$.

A sequence $\left(x_{k}\right)_{k \geq 1} \in(\mathbb{N} \cup\{\infty\})$ is said to be bounded, if there are $l, K \geq 1$ such that $x_{k} \leq K$ for every $k \geq l$. It tends to infinity, if for every $K \geq 1$ there is some $l \geq 1$ such that for every $k \geq l$ we have $x_{k} \geq K$.

Let $\left(X_{k}\right)_{k \geq 1} \in \mathbb{S}$ be a sequence. We define the $\Psi$-limit $\bar{\Psi}$ of $\left(X_{k}\right)_{k \geq 1}$.

L1. If there is an $l \geq 1$ such that $X_{k}=\emptyset$ for every $k \geq l$, then $\bar{\Psi}\left(X_{k}\right)_{k \geq 1}=\infty$. In this case, we call $\left(X_{k}\right)_{k \geq 1}$ an $\infty$-sequence.

For every $z \in\{\curlyvee, \mathbb{X}, \angle\}$, we denote the sequence $\left(\Delta\left(X_{k}, z\right)\right)_{k \geq 1}$ by $\Delta\left(X_{k}, z\right)_{k \geq 1}$. Assume that there is some $l \geq 1$ such that $X_{k} \neq \emptyset$ for every $k \geq l$. In this case, we cannot apply (L1) to $\left(X_{k}\right)_{k \geq 1}$, and we define:

L2. Let $z \in\{\curlyvee, \mathbb{X}, \angle\}$. If $\Delta\left(X_{k}, z\right)_{k \geq 1}$ is bounded, and if for every $z^{\prime}<z$ the sequence $\Delta\left(X_{k}, z^{\prime}\right)_{k \geq 1}$ tends to infinity, then $\bar{\Psi}\left(X_{k}\right)_{k \geq 1}=z$. 
L3. If for every $z \in\{\curlyvee, \mathbb{X}, \angle\}$, the sequence $\Delta\left(X_{k}, z\right)_{k \geq 1}$ tends to infinity, then $\bar{\Psi}\left(X_{k}\right)_{k \geq 1}=\omega$.

If we can apply one of these three definitions to a sequence $\left(X_{k}\right)_{k \geq 1}$, then we call $\left(X_{k}\right)_{k \geq 1}$ a convergent sequence. Otherwise, $\bar{\Psi}\left(X_{k}\right)_{k \geq 1}$ is not defined. We denote the set of all convergent sequences by $\mathfrak{C}(\mathbb{S})$. Every sequence contains a convergent subsequence. Every constant sequence $\left(X_{k}\right)_{k \geq 1}$ is convergent and we have $\bar{\Psi}\left(X_{k}\right)_{k \geq 1}=\Psi\left(X_{1}\right)$. For sequences over $\mathbb{S}$, we define $\cup$ and $\cdot$ componentwise.

Lemma 1. 6] 1. Every subsequence of a convergent sequence is convergent and converges to the same $\Psi$-limit.

2. The set of convergent sequences is closed under componentwise $\cup$ and ·, and $\bar{\Psi}:(\mathfrak{C}(\mathbb{S}), \cup, \cdot) \rightarrow(\mathcal{S}, \min , \cdot)$ is a homomorphism.

The notion of a $\Psi$-limit and a convergent sequence extends naturally to matrices. By Lemma $1(2), \bar{\Psi}: \mathfrak{C}\left(\mathbb{S}_{n \times n}\right) \rightarrow \mathcal{S}_{n \times n}$ is a homomorphism.

For every subset $T \in \mathbb{S}_{n \times n}$ we denote by $\bar{\Psi}\langle T\rangle$ the set of all $\Psi$-limits of all convergent sequences over $\langle T\rangle$. We have $\Psi(\langle T\rangle) \subseteq \bar{\Psi}\langle T\rangle$. We formulate the limitedness problem using $\Psi$-limits.

Proposition 1. 6] Let $\mathcal{A}=\left[Q, E, I, F, E^{<}, E^{\curlyvee}\right]$ be a dd-automaton and denote $T=\theta(\Sigma)$. The following assertions are equivalent:

1. $\mathcal{A}$ is not limited.

2. There is a matrix $a \in \bar{\Psi}\langle T\rangle$ such that $\min \{a[i, j] \mid i \in I, j \in F\}=\omega$.

To give an algorithm for the limitedness problem, we need a method to compute the finite set $\bar{\Psi}\langle T\rangle$ while avoiding to compute the possibly uncountable set $\mathfrak{C}\langle T\rangle$.

\section{The Solution of the Burnside Problem}

We solve the Burnside problem by showing a method to compute the set $\bar{\Psi}\langle T\rangle$. We fuse the approaches of H. LEUNG [10] and the author [5] to the Burnside problems corresponding to distance resp. desert automata. Several new difficulties arise in this fusion, and our proof is more than just a combination of [10] and [5]. By following [510, we use implicitly ideas from I. Simon. This section is a composition of ideas from H. LEUnG, I. Simon, and the author [5/91014, 15[16] together with new ideas.

\subsection{Stabilization}

We define a mapping $\sharp: \mathrm{E}\left(\mathcal{S}_{n \times n}\right) \rightarrow \mathcal{S}_{n \times n}$ which we call stabilization. For every $e \in \mathrm{E}\left(\mathcal{S}_{n \times n}\right)$ and $i, j$ let

$$
e^{\sharp}[i, j]= \begin{cases}\infty & \text { if } e[i, j]=\infty \\ \min \{e[i, l] \cdot e[l, l] \cdot e[l, j] \mid e[l, l]=\curlyvee\} & \text { if there is some } l \\ & \text { such that } e[l, l]=\curlyvee \text { and } \\ & e[i, l], e[l, j] \in\{\curlyvee, \mathbb{M}, \angle\} \\ \omega & \text { otherwise. }\end{cases}
$$

We state some elementary properties of this mapping. 
Remark 1. Let $e \in \mathrm{E}\left(\mathcal{S}_{n \times n}\right)$ and $i, j, l$ be arbitrary.

1. Assume $e[i, l]=\mathbb{M}$ and $e[l, l]=\curlyvee$. Then, $e^{2}[i, l]=\curlyvee$ but $e[i, l]=\mathbb{M}$, i.e., $e \notin \mathrm{E}\left(\mathcal{S}_{n \times n}\right)$. Thus, such $i$ and $l$ cannot exist, and similarly, it is impossible that $e[l, l]=\curlyvee$ and $e[l, j]=\mathbb{M}$.

2. We have $e^{\sharp}[i, j] \neq \mathbb{M}$ by the definition of $e^{\sharp}$. Moreover, we have $e^{\sharp}[i, j]=\infty$ iff $e[i, j]=\infty$.

3. We have $e[i, j]=e^{3}[i, j] \leq e^{\sharp}[i, j]$.

4. If $e[l, l]=\curlyvee$, then we have $e^{\sharp}[l, l]=\curlyvee$ by definition.

5. Assume $e[i, l] \in\{\curlyvee, \angle\}$ and $e[l, l]=\curlyvee$. Then, $e^{\sharp}[i, l]=e[i, l]$. Similarly, if $e[l, l]=\curlyvee$ and $e[l, j] \in\{\curlyvee, \angle\}$ then $e^{\sharp}[l, j]=e[l, j]$.

Now, we state the main result of Section 4. For subsets $M \subseteq \mathcal{S}_{n \times n}$ we define $\langle M\rangle^{\sharp}$ as least subset of $\mathcal{S}_{n \times n}$ which contains $M$ and is closed both under matrix multiplication and stabilization of idempotent matrices.

Theorem 3. Let $T$ be a finite subset of $\mathbb{S}_{n \times n}$. We have $\bar{\Psi}\langle T\rangle=\langle\Psi(T)\rangle^{\sharp}$.

Until Theorem 3 is proved in Section 4.5, we need to establish relations between stabilization and $\Psi$-limits.

\subsection{Stabilization Is a Consistent Mapping}

We establish a first connection between stabilization and $\Psi$-limits of sequences.

Proposition 2. 6] Let $\left(A_{k}\right)_{k \geq 1}$ be a convergent sequence over $\mathbb{S}_{n \times n}$ and let $e=\bar{\Psi}\left(A_{k}\right)_{k \geq 1} \in \mathrm{E}\left(\mathcal{S}_{n \times n}\right)$. Then, $\left(A_{k}^{k}\right)_{k \geq 1}$ is convergent and $\bar{\Psi}\left(A_{k}^{k}\right)_{k \geq 1}=e^{\sharp}$.

The proof is technically involved. It follows the same strategy as the proof of the corresponding proposition in [5]. Our next step is to generalize stabilization from $\mathrm{E}\left(\mathcal{S}_{n \times n}\right)$ to $\operatorname{Reg}\left(\mathcal{S}_{n \times n}\right)$ :

Lemma 2. Stabilization $\sharp$ is a consistent mapping.

Proof. Let $e \in \mathrm{E}\left(\mathcal{S}_{n \times n}\right)$. Let $E \in \mathbb{S}_{n \times n}$ with $\Psi(E)=e$. By Prop. 2] and Lemma[1] $e^{\sharp} e^{\sharp}=\bar{\Psi}\left(E^{k}\right)_{k \geq 1} \bar{\Psi}\left(E^{k}\right)_{k \geq 1}=\bar{\Psi}\left(E^{k} E^{k}\right)_{k \geq 1}=\bar{\Psi}\left(E^{k}\right)_{k \geq 1}=e^{\sharp}$, i.e., $e^{\sharp} \in \mathrm{E}\left(\mathcal{S}_{n \times n}\right)$. Let $a, b \in \mathcal{S}_{n \times n}$ with $a b, b a \in \mathrm{E}\left(\mathcal{S}_{n \times n}\right)$. Let $A, B \in \mathbb{S}_{n \times n}$ with $a=\Psi(A)$, $b=\Psi(B)$. Then, $(a b)^{\sharp}=\bar{\Psi}\left((A B)^{k}\right)_{k \geq 1}=a \bar{\Psi}\left((B A)^{k}\right)_{k \geq 1} b=a(b a)^{\sharp} b$.

Lemma 2 gives a generalization of stabilization to $\operatorname{Reg}\left(\mathcal{S}_{n \times n}\right)$ (cf. Section 3.1). We show three crucial lemmas about stabilization of regular matrices in $\mathcal{S}_{n \times n}$.

Lemma 3. Let $a \in \operatorname{Reg}\left(\mathcal{S}_{n \times n}\right)$ and $i, j$ be arbitrary.

1. If $a[i, j] \in\{\omega, \infty\}$, then $a^{\sharp}[i, j]=a[i, j]$.

2. If $a[i, j]=\curlyvee$, then $a^{\sharp}[i, j] \in\{\curlyvee, \angle, \omega\}$.

3. If $a[i, j] \in\{\mathbb{M}, \angle\}$, then $a^{\sharp}[i, j] \in\{\angle, \omega\}$.

In particular, we have $a^{\sharp}[i, j] \neq \mathbb{M}$ regardless of $a[i, j]$.

Proof (sketch). Let $e \in \mathrm{E}\left(\mathcal{S}_{n \times n}\right)$ with $e=\mathrm{L} a$, i.e., $a=a e, a^{\sharp}=a e^{\sharp}$. The proof follows by an examination of the product $a e^{\sharp}$ and Remark 1. 
The following lemma is an easy adaptation of Lemma 3.9 in [10, p. 107].

Lemma 4. 6] Let $a, b, c \in \mathcal{S}_{n \times n}$ be a smooth product and let $i, j$ such that $(a b c)^{\sharp}[i, j]=\angle$. There are $p, q$ such that we have $a[i, p], c[q, j] \in\{\curlyvee, \mathbb{A}, \angle\}$ and $b[p, q]=b^{\sharp}[p, q]=\curlyvee$. Moreover, we have $a[i, p]=\angle$ or $c[q, j]=\angle$.

Lemma 5. Let $m \geq 1, a_{1}, \ldots, a_{m} \in \mathcal{S}_{n \times n}$ be a smooth product. Let $i, j$ be arbitrary.

1. If $\left(a_{1} \ldots a_{m}\right)^{\sharp}[i, j]=\curlyvee$, then there are $i=i_{0}, \ldots, i_{m}=j$, such that for every $1 \leq l \leq m$, we have $a_{l}\left[i_{l-1}, i_{l}\right]=a_{l}^{\sharp}\left[i_{l-1}, i_{l}\right]=\curlyvee$.

2. If $\left(a_{1} \ldots a_{m}\right)^{\sharp}[i, j]=\angle$, then there are $i=i_{0}, \ldots, i_{m}=j$ such that for every $2 \leq l \leq m-1, a_{l}\left[i_{l-1}, i_{l}\right]=a_{l}^{\sharp}\left[i_{l-1}, i_{l}\right]=\curlyvee$, and $a_{1}\left[i_{0}, i_{1}\right], a_{m}\left[i_{m-1}, i_{m}\right] \in$ $\{\curlyvee, \mathbb{A}, \angle\}$. Moreover, if $m \geq 3$, then $a_{1}\left[i_{0}, i_{1}\right]=\angle$ or $a_{m}\left[i_{m-1}, i_{m}\right]=\angle$.

Proof. (1) Since $\sharp$ is consistent, $\left(a_{1} \ldots a_{m}\right)^{\sharp}=a_{1}^{\sharp} \ldots a_{m}^{\sharp}$. By $a_{1}^{\sharp} \ldots a_{m}^{\sharp}[i, j]=\curlyvee$, there are $i=i_{0}, \ldots, i_{m}=j$ such that for every $1 \leq l \leq m, a_{l}^{\sharp}\left[i_{l-1}, i_{l}\right] \in\{\curlyvee, \mathbb{A}\}$. By Lemma 3, $a_{l}^{\sharp}\left[i_{l-1}, i_{l}\right] \neq \mathbb{A}$, i.e., we have for every $1 \leq l \leq m, a_{l}^{\sharp}\left[i_{l-1}, i_{l}\right]=\curlyvee$. By Lemma 3 in contraposition, we have $a_{l}\left[i_{l-1}, i_{l}\right]=\curlyvee$.

(2) We assume $m \geq 3$, otherwise, the claim is obvious. We apply Lemma 4 to the smooth product $a_{1}\left(a_{2} \ldots a_{m-1}\right) a_{m}$, and (1) to $a_{2} \ldots a_{m-1}$.

\subsection{On the Growth of Entries}

We call a word $w=A_{1} \ldots A_{|w|} \in \mathbb{S}_{n \times n}^{+}$a smooth product if $\Psi\left(A_{1}\right), \ldots, \Psi\left(A_{|w|}\right)$ is a smooth product. We extend the mapping $\Delta$. For a matrix $A \in \mathbb{S}$, a word in $\mathbb{S}_{n \times n}^{+}$, resp. a finite subset $T \subseteq \mathbb{S}_{n \times n}^{+}, \Delta$ yields the maximum among the values on every entry of $A$, of every letter in $w$ of every word $w \in T$ except $\omega$ and $\infty$.

1. For $A \in \mathbb{S}_{n \times n}$, let $\Delta(A)=\max _{i, j, A[i, j] \neq \infty} \Delta(A[i, j])$.

2. For $w=A_{1} \ldots A_{|w|} \in \mathbb{S}_{n \times n}^{+}$, let $\Delta(w)=\max _{l \in\{1, \ldots,|w|\}} \Delta\left(A_{l}\right)$.

3. For $T=\left\{w_{1}, \ldots, w_{|T|}\right\} \subseteq \mathbb{S}_{n \times n}^{+}$, let $\Delta(T)=\max _{l \in\{1, \ldots,|T|\}} \Delta\left(w_{l}\right)$.

We extend $\Delta^{\prime}$ from Section 3.4 in the same way.

Proposition 3. Let $w \in \mathbb{S}_{n \times n}^{+}$be a smooth product and $i, j$ be arbitrary.

1. If $\Psi(w)^{\sharp}[i, j]=\curlyvee$, then $\Delta^{\prime}(\alpha(w)[i, j]) \leq 2 \Delta^{\prime}(w)$, i.e., $\Delta(\alpha(w)[i, j]) \leq 2 \Delta^{\prime}(w)$.

2. If $\Psi(w)^{\sharp}[i, j]=\omega$, then $\Delta(\alpha(w)[i, j]) \geq \frac{\sqrt{|w|}}{5^{n^{2} n}}-1$.

Proof. We denote $w=A_{1} \ldots A_{|w|}$ and $a_{l}=\Psi\left(A_{l}\right)$ for every $l \in\{1, \ldots,|w|\}$.

We show (1). By Lemma [5(1), there are $i=i_{0}, \ldots, i_{|w|}=j$ satisfying for every $l \in\{1, \ldots,|w|\}, a_{l}\left[i_{l-1}, i_{l}\right]=\curlyvee$. Thus, every $A\left[i_{l-1}, i_{l}\right]$ contains a $\pi_{l}$ with $\Psi\left(\pi_{l}\right)=\curlyvee$ and $\Delta\left(\pi_{l}\right) \leq \Delta^{\prime}(w)$, i.e., $\Delta^{\prime}(\alpha(w)[i, j]) \leq \Delta\left(\pi_{1} \ldots \pi_{|w|}\right) \leq 2 \cdot \Delta^{\prime}(w)$.

We sketch (2). By contradiction, let $\pi \in \alpha(w)[i, j]$ with $\Delta(\pi)<\frac{\sqrt{|w|}}{5^{n^{2} n}}-1$. By various counting arguments, we show some $1 \leq q<r \leq|w|$ and some $l$ such that 
$\pi \in \alpha\left(A_{1} \ldots A_{q}\right)[i, l] \cdot \alpha\left(A_{q+1} \ldots A_{r}\right)[l, l] \cdot \alpha\left(A_{r+1} \ldots A_{|w|}\right)[l, j]$. We can choose $q$ and $r$ such that $a_{q+1} \ldots a_{r}$ is an idempotent. We can factorize $\pi=\pi_{1} \pi_{2} \pi_{3}$ such that $\pi_{2} \in \alpha\left(A_{q+1} \ldots A_{r}\right)[l, l]$. By $\Delta(\pi)<\frac{\sqrt{|w|}}{5^{n^{2} n}}-1$, we can choose $q$ and $r$ such that $\Psi\left(\pi_{2}\right)=\curlyvee$, i.e., $\left(a_{q+1} \ldots a_{r}\right)[l, l]=\curlyvee$. Then, $\left(a_{q+1} \ldots a_{r}\right)^{\sharp}[l, l]=\curlyvee$, and we can conclude $\Psi(w)^{\sharp}[i, j] \in\{\curlyvee, \angle\}$.

In [6], we use Lemma [5 2) to show a corresponding proposition for the case $\Psi(w)^{\sharp}[i, j]=\angle$ and we obtain the following corollary.

Corollary 1. [6] Let $w \in \mathbb{S}_{n \times n}^{+},|w| \geq 3$ be a smooth product and $i, j$ be arbitrary. Let $z=\Psi(w)^{\sharp}[i, j]$.

1. If $z \in\{\curlyvee, \angle\}$, then there is a path $\pi \in \alpha(w)[i, j]$ satisfying $\Psi(\pi)=z$ and $\Delta(\pi) \leq 4 \Delta^{\prime}(w)$.

2. For every $\pi \in \alpha(w)[i, j]$ with $\Psi(\pi)<z$, we have $\Delta(\pi) \geq \frac{\sqrt{|w|}}{5^{n^{2} n}}-1$.

Note that $\Psi(\pi)<z$ in (2) is the ordering $\curlyvee<\mathbb{M}<\angle<\ldots$ of $\mathcal{S}$.

\subsection{Stabilization of Word Matrices}

For matrices $A \in \mathbb{S}_{n \times n}$, we define $A^{\sharp}$ if $\Psi(A) \in \operatorname{Reg}\left(\mathcal{S}_{n \times n}\right)$. Let $a=\Psi(A)$.

$$
A^{\sharp}[i, j]= \begin{cases}A[i, j] & \text { if } a[i, j]=a^{\sharp}[i, j] \\ \omega & \text { if } a[i, j] \neq a^{\sharp}[i, j]=\omega \\ \{\pi \in A[i, j] \mid \Psi(\pi)=\angle\} & \text { if } a[i, j] \neq a^{\sharp}[i, j]=\angle .\end{cases}
$$

By Lemma 3, the cases in this definition are complete. Intuitively, the definition of $A^{\sharp}$ simply means to apply all the changes between $a$ and $a^{\sharp}$ to $A$. It seems that we have $\Psi\left(A^{\sharp}\right)=a^{\sharp}$. However, one can construct counterexamples in which $a[i, j] \neq a^{\sharp}[i, j]=\angle$, but $A[i, j]$ does not contain some word $\pi$ with $\Psi(\pi)=\angle$. In this case, $A^{\sharp}[i, j]=\emptyset$, i.e., $\Psi\left(A^{\sharp}\right)=\infty \neq \angle=a^{\sharp}[i, j]$. On the other hand, if $a[i, j] \neq a^{\sharp}[i, j]=\angle$ and $A$ is the result of a smooth product of 3 matrices, then there is by Corollary $\mathbb{1}(1)$ some $\pi \in A[i, j]$ with $\Psi(\pi)=\angle$.

Lemma 6. 6] Let $w \in \mathbb{S}_{n \times n}^{+}$be a smooth product with $|w| \geq 3$. We have $\Psi\left(\alpha(w)^{\sharp}\right)=\Psi(\alpha(w))^{\sharp}$.

We define a relation to compare word matrices. Let $K \geq 1$ and $X, Y \in \mathbb{S}$. We write $X \preceq_{K} Y$ if we can transform $X$ into $Y$ by removing words $\pi$ with $\Delta(\pi)>K$ from $X$. More precisely, we write $X \preceq_{K} Y$ if we have:

1. If $X=\emptyset$, then $Y=\emptyset$.

2. If $X \neq \emptyset$, then $X \supseteq Y \neq \emptyset$.

3. $X$ and $Y$ "agree in their bounded words", i.e., $\{\pi \in X \mid \Delta(\pi) \leq K\} \subseteq Y$.

If $X$ contains some word, then we do not have $X \preceq_{K} \emptyset$, regardless of whether there are words $\pi \in X$ with $\Delta(\pi) \leq K$. However, if $X$ contains some word, but we have $\Delta(\pi)>K$ for every $\pi \in X$, then we have $X \preceq_{K} \omega$.

We generalize $\preceq_{K}$ componentwise to matrices in $\mathbb{S}_{n \times n}$. It is easy to show that $\preceq_{K}$ on $\mathbb{S}$ is stable w.r.t. $\cup$ and , i.e., $\preceq_{K}$ on $\mathbb{S}_{n \times n}$ is stable w.r.t. multiplication. 
Lemma 7. Let $K \geq 1$, and let $w \in \mathbb{S}_{n \times n}^{+}$be a smooth product with $|w| \geq\left(5^{n^{2}} n(K+2)\right)^{2}$. We have $\alpha(w) \preceq_{K} \alpha(w)^{\sharp}$.

Proof. Denote $w=A_{1} \ldots A_{|w|}$, and let $A=\alpha(w)$ and $a=\Psi(A)$. Let $i, j$ be arbitrary. We consider the cases in the definition of $A^{\sharp}$.

If $a[i, j]=a^{\sharp}[i, j]$, then $A[i, j]=A^{\sharp}[i, j]$ and in particular $A[i, j] \preceq_{K} A^{\sharp}[i, j]$.

Assume $a[i, j] \neq a^{\sharp}[i, j]=\omega$. Then, $A^{\sharp}[i, j]=\omega$. By Lemma 3, we have $a[i, j] \neq \infty$, i.e., $A[i, j] \neq \emptyset$ which shows $(1,2)$ in the definition of $\preceq_{k}$. In order to verify (3), we have to show for every $\pi \in X, \Delta(\pi)>K$. This follows from Corollary 11(2) and the length assumption on $w$.

We can deal with the case $a[i, j] \neq a^{\sharp}[i, j]=\angle$ in the same way.

\subsection{The Proof of Theorem 3}

We show a proposition which allows to transform words in $\mathbb{S}_{n \times n}^{+}$. We will use it to transform words in sequences $\left(w_{k}\right)_{k>1} \in T^{+}$to examine the $\Psi$-limit of the sequence $\left(\alpha\left(w_{k}\right)\right)_{k \geq 1}$ in order to show $\bar{\Psi}\langle T\rangle \subseteq\langle\Psi(T)\rangle^{\sharp}$.

Proposition 4. Let $K \geq 2$. Let $T$ be some finite subset of $\mathbb{S}_{n \times n}$. There is some $x_{K}>K$ such that for every $w \in T^{+}$there is a $B \in \mathbb{S}_{n \times n}$ satisfying $\Psi(B) \in\langle\Psi(T)\rangle^{\sharp}, \alpha(w) \preceq_{K} B$, and $\Delta^{\prime}(B) \leq x_{K}$.

We should pay some attention to the conditions $\Delta(B) \leq x_{K}$ and $\alpha(w) \preceq_{K} B$. Let $i, j$ be arbitrary. If $\alpha(w)[i, j] \in\{\omega, \emptyset\}$, then $\alpha(w)[i, j]=B[i, j]$.

If we have $\Delta(\alpha(w)[i, j]) \leq K$, then $\alpha(w)[i, j] \supseteq B[i, j]$ but $\alpha(w)[i, j]$ and $B[i, j]$ agree in their bounded words (cf. 2,3 in the definition of $\preceq_{K}$ ).

If $\Delta(\alpha(w)[i, j])>x_{K}$, then $\alpha(w) \preceq_{K} B$ and $\Delta(B) \leq x_{K}$ imply $B[i, j]=\omega$.

However, if $K<\Delta(\alpha(w)[i, j]) \leq x_{K}$, then we do not know whether we have $\Delta(B[i, j]) \leq x_{K}$ or $\Delta(B[i, j])=\omega$. We avoid this problem by applying Prop. 4 just on words $w$ for which either $\Delta(\alpha(w)[i, j]) \leq K$ or $x_{K}<\Delta(\alpha(w)[i, j])$.

If we cut the assertion $\Delta^{\prime}(B) \leq x_{K}$ in Prop. 4 then we can prove it by setting $B=\alpha(w)$ and $x_{K}=K$. In fact, the proof of Prop. 4 is essentially based on the idea to define $B$ as $\alpha(w)$ with some adjustments in a way that these adjustments keep the properties $\Psi(B) \in\langle\Psi(T)\rangle^{\sharp}$ and $\alpha(w) \preceq_{K} B$.

If we assume in Prop. 4 that $w$ is a long smooth product, then we can prove it by setting $B=\alpha(w)^{\sharp}$ and $x_{k}=4 \Delta^{\prime}(T)$. By Lemma 6, we achieve $\Psi(B)=$ $\Psi\left(\alpha(w)^{\sharp}\right)=\Psi(\alpha(w))^{\sharp}$ which belongs to $\langle\Psi(T)\rangle^{\sharp}$ because $\Psi(\alpha(w)) \in\langle\Psi(T)\rangle^{\sharp}$. We have $\alpha(w) \preceq_{K} B$ by Lemma 7 , and we get $\Delta^{\prime}(B) \leq 4 \Delta^{\prime}(T)$ by Corollary $1(1)$.

We establish the following lemma to prove Prop. 4

Lemma 8. Let $K \geq 2$ and $x \geq 1$ be arbitrary. Let $I^{\prime} \subsetneq I \subseteq\langle\Psi(T)\rangle^{\sharp}$ be two ideals of $\langle\Psi(T)\rangle^{\sharp}$ such that $I \backslash I^{\prime}$ is a J-class of $\langle\Psi(T)\rangle^{\sharp}$.

There is some $x^{\prime} \geq 1$ such that for every $w=A_{1} \ldots A_{|w|} \in \mathbb{S}_{n \times n}^{+}$satisfying

A1. $\Psi\left(A_{1}\right), \ldots, \Psi\left(A_{|w|}\right) \in\langle\Psi(T)\rangle^{\sharp}$,

A2. $\Delta^{\prime}(w) \leq x$,

A3. For every $1 \leq l \leq|w|-1, \Psi\left(A_{l} A_{l+1}\right) \in I$, 
there is some $v=B_{1} \ldots B_{|v|} \in \mathbb{S}_{n \times n}^{+}$satisfying $\alpha(w) \preceq_{K} \alpha(v)$ and

C1. $\Psi\left(B_{1}\right), \ldots, \Psi\left(B_{|v|}\right) \in\langle\Psi(T)\rangle^{\sharp}$,

C2. $\Delta^{\prime}(v) \leq x^{\prime}$,

C3. For every $1 \leq l \leq|v|-1, \Psi\left(B_{l} B_{l+1}\right) \in I^{\prime}$.

In particular, this assertion is true for $x^{\prime}=2\left(5^{n^{2}} n(K+2)\right)^{2} x$.

At first, note the similarity between the assumptions (A1, A2, A3) and the claims (C1, C2, C3). This similarity enables us to apply Lemma 8 inductively on a chain of ideals $\emptyset \subsetneq \ldots \subsetneq I_{2} \subsetneq I_{1} \subseteq\langle\Psi(T)\rangle^{\sharp}$ to prove Prop. 4. In the first step of the induction, we simply set $x=\Delta^{\prime}(T)$ and $I=\langle\Psi(T)\rangle^{\sharp}$, and for every $w \in T^{+}$ (A1, A2, A3) are obviously satisfied. In the last step of this induction, $I^{\prime}=\emptyset$, and thus, (C3) implies that $v$ has the length 1 , and $v$ is the matrix $B$ which we require to prove Prop. 4 See [6] for the details of the proof of Prop. 4 .

We are not interested in whether $A_{1}, \ldots, A_{|w|}, B_{1}, \ldots, B_{|v|}$ belong to $\langle T\rangle$, we just assume resp. show that their images under $\Psi$ belong to $\langle\Psi(T)\rangle^{\sharp}$.

Proof (Lemma 8). Let $K, x$, and $w=A_{1} \ldots A_{|w|} \in \mathbb{S}_{n \times n}^{+}$as in the lemma. We factorize $w$ into words $v_{1}, v_{2}, \ldots, v_{m}$. If $\Psi\left(A_{1}\right) \in I^{\prime}$, then let $v_{1}=A_{1}$ and proceed with $A_{2} \ldots A_{|w|}$. If $\Psi\left(A_{1}\right) \notin I^{\prime}$, then let $v_{1}$ be the longest prefix of $w$ satisfying $\Psi\left(v_{1}\right) \notin I^{\prime}$ and proceed with the remaining part of $w$. If $\left|v_{l}\right|>1$ for an $1 \leq l \leq m$, then $\Psi\left(v_{l}\right) \in I \backslash I^{\prime}$, since $\Psi\left(v_{l}\right) \notin I^{\prime}$ by construction and $\Psi\left(v_{l}\right) \in I$ by (A3). We get an $m \geq 1$ and $v_{1}, \ldots, v_{m} \in \mathbb{S}_{n \times n}^{+}$such that

1. $A_{1} \ldots A_{|w|}=v_{1} \ldots v_{m} \quad$ (concatenation of words)

2. $\Psi\left(v_{1}\right), \ldots, \Psi\left(v_{m}\right) \in\langle\Psi(T)\rangle^{\sharp}$

3. For every $1 \leq l \leq m-1, \Psi\left(\alpha\left(v_{l} v_{l+1}\right)\right) \in I^{\prime}$. (by construction of $\left.v_{l}\right)$

4. For every $1 \leq l \leq m$ with $\left|v_{l}\right|>1$, we have $\Psi\left(\alpha\left(v_{l}\right)\right) \in I \backslash I^{\prime}$.

Let $1 \leq l \leq m$ be arbitrary.

Case 1: $\left|v_{l}\right|<2\left(5^{n^{2}} n(K+2)\right)^{2}$

We set $B_{l}=\alpha\left(v_{l}\right)$. Then, $\alpha\left(v_{l}\right) \preceq_{K} B_{l}$ and $B_{l}$ satisfies (C1). Moreover, $\Delta^{\prime}\left(B_{l}\right) \leq\left|v_{l}\right| \cdot \Delta^{\prime}\left(v_{l}\right) \leq 2\left(5^{n^{2}} n(K+2)\right)^{2} x=x^{\prime}$, i.e., $B_{l}$ satisfies (C2).

Case 2: $\left|v_{l}\right| \geq 2\left(5^{n^{2}} n(K+2)\right)^{2}$

We denote $v_{l}$ as $v_{l}=V_{1} \ldots V_{\left|v_{l}\right|}$. We transform $v_{l}$ into a word $u$. If $\left|v_{l}\right|$ is even, then we set $u=\alpha\left(V_{1} V_{2}\right) \alpha\left(V_{3} V_{4}\right) \ldots \alpha\left(V_{\left|v_{l}\right|-1} V_{\left|v_{l}\right|}\right)$. Otherwise, $u=$ $\alpha\left(V_{1} V_{2}\right) \alpha\left(V_{3} V_{4}\right) \ldots \alpha\left(V_{\left|v_{l}\right|-2} V_{\left|v_{l}\right|-1} V_{\left|v_{l}\right|}\right)$. Clearly, $\alpha\left(v_{l}\right)=\alpha(u)$.

We have $|u| \geq\left(5^{n^{2}} n(K+2)\right)^{2}$. We denote the letters of $u$ by $u=U_{1} \ldots U_{|u|}$. Let $1 \leq k \leq|u|$. By $(\mathrm{A} 3), \Psi\left(U_{k}\right) \in I$. If $\Psi\left(U_{k}\right) \in I^{\prime}$, then $\Psi(\alpha(u)) \in I^{\prime}$ and $\Psi(\alpha(u))=\Psi\left(\alpha\left(v_{l}\right)\right) \in I^{\prime}$ which contradicts (4), above. Hence, $\Psi\left(U_{k}\right) \in I \backslash I^{\prime}$. Consequently, $I \backslash I^{\prime}$ is a regular J-class of $\langle\Psi(T)\rangle^{\sharp}$, and $u$ is a smooth product. Hence, we can define $B_{l}=\alpha(u)^{\sharp}=\alpha\left(v_{l}\right)^{\sharp}$.

By Lemma 6, $\Psi\left(B_{l}\right)=\Psi\left(\alpha(u)^{\sharp}\right)=\Psi(\alpha(u))^{\sharp}$. By $\Psi(\alpha(u)) \in\langle\Psi(T)\rangle^{\sharp}$, we have $\Psi(\alpha(u))^{\sharp} \in\langle\Psi(T)\rangle^{\sharp}$. To sum up, we have $\Psi\left(B_{l}\right)=\Psi(\alpha(u))^{\sharp} \in\langle\Psi(T)\rangle^{\sharp}$. By Lemma 7 , we have $\alpha(u) \preceq_{K} \alpha(u)^{\sharp}$, i.e., $\alpha\left(v_{l}\right) \preceq_{K} B_{l}$.

Let $i, j$ be arbitrary. If $B_{l}[i, j]$ contains some word, then $\Psi\left(B_{l}\right)[i, j] \in\{\curlyvee, \angle\}$. By Corollary[1(1), $\Delta^{\prime}\left(B_{l}[i, j]\right) \leq 4 \Delta^{\prime}(u) \leq 12 \Delta^{\prime}(w) \leq 12 x<x^{\prime}$ (C2). 
We show (C3). By (3) above, $\Psi\left(v_{l}\right) \Psi\left(v_{l+1}\right) \in I^{\prime}$ for every $l \in\{1, \ldots, m-1\}$. In both case 1 and 2 , we have $\Psi\left(B_{l}\right) \leq_{\mathrm{L}} \Psi\left(v_{l}\right)$ and $\Psi\left(B_{l+1}\right) \leq_{\mathrm{R}} \Psi\left(v_{l+1}\right)$, i.e., $\Psi\left(B_{l}\right) \Psi\left(B_{l+1}\right) \leq_{\mathrm{J}} \Psi\left(v_{l}\right) \Psi\left(v_{l+1}\right) \in I^{\prime}$, i.e., $\Psi\left(B_{l}\right) \Psi\left(B_{l+1}\right)=\Psi\left(B_{l} B_{l+1}\right) \in I^{\prime}$.

In both case 1 and 2 , we have seen $\alpha\left(v_{l}\right) \preceq_{K} \alpha\left(B_{l}\right)$ for every $1 \leq l \leq m$. By the stability of $\preceq_{K}$ w.r.t. matrix multiplication it follows $\alpha(w) \preceq_{K} \alpha(v)$.

Proof (Theorem [3). We show $\langle\Psi(T)\rangle^{\sharp} \subseteq \bar{\Psi}\langle T\rangle$. We have $\Psi(T) \subseteq \bar{\Psi}\langle T\rangle$, because for every $A \in T, \Psi(A)$ is the $\Psi$-limit of $(A)_{k \geq 1}$. Moreover, $\bar{\Psi}\langle T\rangle$ is closed under multiplication (Lemma 1) and stabilization of idempotents (Prop. 1).

We show $\bar{\Psi}\langle T\rangle \subseteq\langle\Psi(T)\rangle^{\sharp}$. Let $\left(w_{k}\right)_{k \geq 1} \in T^{+}$be some sequence such that $\alpha\left(w_{k}\right)_{k \geq 1}$ is convergent. Let $a=\bar{\Psi}\left(\alpha\left(w_{k}\right)\right)_{k \geq 1}$. We have to show $a \in\langle\Psi(T)\rangle^{\sharp}$.

By subsequence selection arguments, we can assume some bound $K \geq 1$ such that we have for every $l \geq 1, z \in\{\curlyvee, \mathbb{A}, \angle\}$ and every $i, j$ :

1. If $a[i, j]=\infty$, then $\alpha\left(w_{l}\right)[i, j]=\infty$.

2. If $\Delta\left(\alpha\left(w_{k}\right)[i, j], z\right)_{k \geq 1}$ is bounded, then $\Delta\left(\alpha\left(w_{l}\right)[i, j], z\right) \leq K$.

Let $x_{K}$ be provided by Prop. 4. There is a word $w$ among $\left(w_{k}\right)_{k \geq 1}$ such that:

3. If $\Delta\left(\alpha\left(w_{k}\right)[i, j], z\right)_{k \geq 1}$ tends to infinity, then $\Delta(\alpha(w)[i, j], z)_{k \geq 1}>x_{K}$.

By Prop. 4 on $w$ we obtain some $B \in \mathbb{S}_{n \times n}$. We have $\Psi(B) \in\langle\Psi(T)\rangle^{\sharp}$. In the rest of the proof, we show $a=\Psi(B)$ and $a \in\langle\Psi(T)\rangle^{\sharp}$ follows. Let $i, j$ be arbitrary.

If $a[i, j]=\infty$, then we have by $\alpha(w)[i, j]=\infty$ due to $(1)$ and $\alpha(w) \preceq_{K} B$ (Prop. [4), $B[i, j]=\infty$, i.e., $\Psi(B[i, j])=\infty=a[i, j]$.

Assume $a[i, j]=\angle$. Then, $\Delta\left(\alpha\left(w_{k}\right), \angle\right)_{k>1}$ is bounded. By (2) above, there is a $\pi \in \alpha(w)[i, j]$ with $\Psi(\pi)=\angle$ and $\Delta(\pi) \leq K$. By $\alpha(w) \preceq_{K} B, \pi \in B[i, j]$, i.e, $\Psi(B[i, j]) \in\{\curlyvee, \mathbb{A}, \angle\}$. By contradiction, assume $\Psi(B[i, j])=\curlyvee$. From all words $\pi \in B[i, j]$ with $\Psi(\pi)=\curlyvee$ choose a word $\pi$ for which $\Delta(\pi)$ is minimal. By $\alpha(w) \preceq_{K} B, \pi \in \alpha(w)[i, j]$. Because $\Delta\left(\alpha\left(w_{k}\right), \curlyvee\right)_{k \geq 1}$ tends to infinity, we have by $(3)$ above $\Delta(\pi)>x_{K}$, and we can conclude $\Delta^{\prime}(B)>x_{K}$, which is a contradiction. Hence, $\Psi(B[i, j]) \neq \curlyvee$. We can show $\Psi(B[i, j]) \neq M$ in the same way. To sum up, $\Psi(B[i, j])=\angle=a[i, j]$.

We deal with the cases $a[i, j] \in\{\curlyvee, \mathbb{M}, \omega\}$ in the same way [6].

\section{Proof (Theorem 11). We combine Prop. 1 and Theorem 3 .}

\section{On the Star Height One Problem}

Let $\Sigma$ be an alphabet. Every word $w \in \Sigma^{*}$ is a rational expression of star height 0 , i.e., $\operatorname{sh}(w)=0$. If $r$ and $s$ are rational expressions over $\Sigma^{*}$, then $r s$ and $r \cup s$ are rational expressions of star height $\max \{\operatorname{sh}(r), \operatorname{sh}(s)\}$, but $r^{*}$ is of star height $\operatorname{sh}(r)+1$. The star height of a language $L$ is denoted by $\operatorname{sh}(L)$ and defined as the minimum of $\operatorname{sh}(r)$ over all rational expressions $r$ such that $L(r)=L$.

A language $L$ is of star height 0 iff it is finite. Already for $\Sigma=\{a, b\}$, there are languages of arbitrary star height 1]. For example, $a b^{*}, b a^{*} b^{*} a a \cup a^{*}$ and $\left(a^{*} b\right)^{*}=\varepsilon \cup\{a, b\}^{*} b$ are of star height 1 , but $\left(a^{*} b^{*} c\right)^{*}$ is of star height 2 . 
The star height 1 problem is to decide whether a given recognizable language $L$ is of star height one. It was raised in a more general way by L.C. EGGAN in 1963 [2]. Because $\operatorname{sh}(L)=0$ is easily decidable, the star height 1 problem is equivalent to the question whether $\operatorname{sh}(L) \leq 1$. In 1982, K. HAsHiguCHI showed that such an algorithm exists [4]. He showed in an involved proof that the language of an automaton $\mathcal{A}$ is of star height one iff $L(\mathcal{A})=L(r)$ for a rational expression $r$ of a certain size with $\operatorname{sh}(r)=1$. The "certain size" is a very large bound which depends on the number of states of $\mathcal{A}$.

Here, we give a new solution to the star height one problem by a reduction to the limitedness problem for dd-automata. This provides a much better, although still very large bound for the complexity.

\subsection{Normal Forms of Rational Expressions}

Let $n \in \mathbb{N}$. A rational expression $r$ is in single string form if $r$ is of the form $r=a_{1} K_{1}^{*} a_{2} K_{2}^{*} \ldots a_{n} K_{n}^{*}$ where for every $1 \leq i \leq n, a_{i} \in \Sigma$ and $K_{i} \in \Sigma^{+}$is finite. We call $n$ the length of $r$. The length of a longest word in the sets $K_{i}$ is called the degree of $r$ and denoted by $d(r)$. The empty word is a rational expression in string form of length and degree 0. A rational expression $s$ is in string form if $s=s_{1} \cup \cdots \cup s_{k}$ for a $k \geq 1$ where each $s_{i}$ is a rational expression in single string form. The degree of $s$ is the maximum of the degrees of $s_{1}, \ldots, s_{k}$.

We can transform every rational expression $r$ with $\operatorname{sh}(r) \leq 1$ into an equivalent expression in string form by using the distributivity of concatenation over union and inserting $\emptyset^{*}$, e.g., we transform $a b\{a b\}^{*} a$ into $a \emptyset^{*} b\{a b\}^{*} a \emptyset^{*}[6]$.

For the rest of the paper, let $L \subseteq \Sigma^{*}$ be a language which is recognized by a deterministic automaton $\mathcal{A}=\left[Q, \delta, q_{I}, F\right]$. It is of crucial importance that $\delta: Q \times \Sigma \rightarrow Q$ is total! We extend $\delta$ to $\delta: \mathcal{P}(Q) \times \Sigma^{*} \rightarrow \mathcal{P}(Q)$ as usual.

Let $n \geq 0$. A sequence $P_{0}, \ldots, P_{n} \in \mathcal{P}_{n e}(Q)$ is called a single syntactic expression from $P_{0}$ to $P_{n}$ of length $n$. Every finite set of single syntactic expression is called a syntactic expression.

Let $d \geq 1$. For every $P, R \in \mathcal{P}_{n e}(Q)$, let

1. $S_{d}(P)=\{\varepsilon\}$, and

2. $S_{d}(P, R)=\{a \in \Sigma \mid \delta(P, a) \subseteq R\}\left\{w \in \Sigma^{+}|\delta(R, w) \subseteq R,| w \mid \leq d\right\}^{*}$.

We define $S_{d}\left(P_{0}, \ldots, P_{n}\right)=S_{d}\left(P_{0}, P_{1}\right) S_{d}\left(P_{1}, P_{2}\right) \ldots S_{d}\left(P_{n-1}, P_{n}\right)$ for every $P_{0}, \ldots, P_{n} \in \mathcal{P}_{n e}(Q)$. For every syntactic expression $T$, let $S_{d}(T)=\cup_{t \in T} S_{d}(t)$.

Let $d \geq 1$ and $P, R \in \mathcal{P}_{n e}(Q)$ be arbitrary. We can easily show $S_{d}(P, R, R)=$ $S_{d}(P, R) S_{d}(R, R) \subseteq S_{d}(P, R)$. For every single syntactic expression $t$ from $P$ to $R$, and every $w \in S_{d}(t)$, we have $\delta(P, w) \subseteq R$.

Let $d, n \geq 1$. We denote by $\varrho_{n}(\mathcal{A})$ the union of all single syntactic expressions from $\left\{q_{I}\right\}$ to subsets of $F$ of a length of at most $n$. For every $w \in S_{d}\left(\varrho_{n}(\mathcal{A})\right)$, we have $\delta\left(q_{I}, w\right) \in F$. Because $\delta$ is total, we have $w \in L$, i.e., $S_{d}\left(\varrho_{n}(\mathcal{A})\right) \subseteq L$.

Proposition 5. There are $d, n \geq 1$ with $L=S_{d}\left(\varrho_{n}(\mathcal{A})\right)$ iff $\operatorname{sh}(L) \leq 1$.

Proof (sketch). . . $\Rightarrow$. For given $d, n \geq 1$, we can easily construct a rational expression $r$ with $\operatorname{sh}(r) \leq 1$ such that $L(r)=S_{d}\left(\varrho_{n}(\mathcal{A})\right)=L$. 
$\ldots \Leftarrow \ldots$ We have $L=L(r)$ for an expression $r$ in string form. We consider the case that $r=a_{1} K_{1}^{*} \ldots a_{n} K_{n}^{*}$. Let $d$ be the degree of $r$. Let $P_{0}=\left\{q_{I}\right\}$, and for $1 \leq i \leq n$, let $P_{i}=\delta\left(P_{i-1}, a_{i} K_{i}^{*}\right)$. We have $L(r) \subseteq S_{d}\left(P_{0}, \ldots, P_{n}\right)$. By $L(r) \subseteq L$, the inclusion $P_{n} \subseteq F$ holds. Thus, $P_{0}, \ldots, P_{n}$ belongs to $\varrho_{n}(\mathcal{A})$, and hence, $L=L(r) \subseteq S_{d}\left(\varrho_{n}(\mathcal{A})\right) \subseteq L$.

\subsection{A Reduction the Limitedness Problem for dd-Automata}

We show the decidability of the existence of $d, n \geq 1$ with $L=S_{d}\left(\varrho_{n}(\mathcal{A})\right)$. By following a similar approach as K. HASHIGUCHI [3]4, we can construct for each given $d \in \mathbb{N}$ a distance automaton $\mathcal{A}_{d}$ which is limited iff there is some $n$ such that $L=S_{d}\left(\varrho_{n}(\mathcal{A})\right)$. Conversely, we can construct for each $n \in \mathbb{N}$ a desert automaton $\mathcal{A}_{n}^{\prime}$ which is limited iff there is some $d$ such that $L=S_{d}\left(\varrho_{n}(\mathcal{A})\right)$.

We construct a dd-automaton $\mathcal{A}^{\prime}$ which is limited iff there are $d, n \geq 1$ with $L=S_{d}\left(\varrho_{n}(\mathcal{A})\right)$. In 6, we explain this construction for the language $\left(a \cup b^{*} c\right)^{*}$. Let $Q^{\prime}=q_{I}^{\prime} \cup\left(\mathcal{P}_{n e}(Q) \times \mathcal{P}_{n e}(Q)\right)$. We define the transitions $E^{\prime}$, péages $E^{\prime<}$, and water transitions $E^{\prime \curlyvee}$ as follows. Let $a \in \Sigma$.

1. For every $P, R \in \mathcal{P}_{n e}(Q),((P, P), a,(R, R))$ is a transition of $\mathcal{A}^{\prime}$ iff $P \neq R$ and $\delta(P, a) \subseteq R$. This transition is both a water transition and a péage.

Moreover, for every $R \in \mathcal{P}_{n e}(Q),\left(q_{I}^{\prime}, a,(R, R)\right)$ is a transition of $\mathcal{A}^{\prime}$ iff $\delta\left(q_{I}, a\right) \subseteq R$. This transition is both a water transition and a péage.

2. For every $P_{1}, P_{2}, R \in \mathcal{P}_{n e}(Q),\left(\left(P_{1}, R\right), a,\left(P_{2}, R\right)\right)$ is a transition of $\mathcal{A}^{\prime}$ iff $\delta\left(P_{1}, a\right) \subseteq P_{2}$. It is not a péage. It is a water transition iff $P_{2}=R$.

This construction is closely related to our notion of syntactic expressions: let $d \geq 1$ and $P_{1}, P_{2} \in \mathcal{P}_{n e}(Q)$ with $P_{1} \neq P_{2}$. Choose some arbitrary $w \in S_{d}\left(P_{1}, P_{2}\right)$. We factorize $w$ according to the definition of $S_{d}\left(P_{1}, P_{2}\right)$. There are some $a \in \Sigma$, $k \geq 0$, and $w_{1}, \ldots, w_{k} \in \Sigma^{+}$such that $w=a w_{1} \ldots w_{k}$. Moreover, we can assume $\delta\left(P_{1}, a\right) \subseteq P_{2}$ and for every $1 \leq i \leq k, \delta\left(P_{2}, w_{i}\right) \subseteq P_{2}$ and $\left|w_{i}\right| \leq d$. The automaton $\mathcal{A}^{\prime}$ can read $a$ from $\left(P_{1}, P_{1}\right)$ to $\left(P_{2}, P_{2}\right)$. The transition $\left(\left(P_{1}, P_{1}\right), a,\left(P_{2}, P_{2}\right)\right)$ is both a péage and a water transition. For every $1 \leq i \leq k, \mathcal{A}^{\prime}$ can read $w_{i}$ in a loop at $\left(P_{2}, P_{2}\right)$ by visiting only states in $\mathcal{P}_{n e}(Q) \times\left\{P_{2}\right\}$. The last transition is a water transition. In this way, $\mathcal{A}^{\prime}$ can read $w=a w_{1} \ldots w_{k}$ from $\left(P_{1}, P_{1}\right)$ to $\left(P_{2}, P_{2}\right)$ along some path $\pi$ such that $\pi$ contains exactly one péage and $\pi$ does not contain $d$ consecutive non-water transitions.

Now, let $P_{3} \in \mathcal{P}_{n e}(Q)$ with $P_{2} \neq P_{3}$, and choose some $w^{\prime} \in S_{d}\left(P_{1}, P_{2}, P_{3}\right)$. By following the same idea, $\mathcal{A}^{\prime}$ can read $w^{\prime}$ from $\left(P_{1}, P_{1}\right)$ to $\left(P_{3}, P_{3}\right)$ along some path $\pi^{\prime}$ such that $\pi^{\prime}$ contains exactly two péages and $\pi^{\prime}$ does not contain $d$ consecutive non-water transitions.

We complete the definition of $\mathcal{A}^{\prime}$ by defining the set of accepting states as $F^{\prime}=\left\{(R, R) \mid R \in \mathcal{P}_{n e}(F)\right\}$. If $\varepsilon \in L$, then $q_{I}^{\prime}$ is also an accepting state. We denote the mapping of $\mathcal{A}^{\prime}$ by $\Delta$.

Let $P_{1}, R_{1}, P_{2}, R_{2} \in \mathcal{P}_{n e}(Q)$. If there is a path from $\left(P_{1}, R_{1}\right)$ to $\left(P_{2}, R_{2}\right)$ in $\mathcal{A}^{\prime}$ with some label $w$, then $\delta\left(P_{1}, w\right) \subseteq P_{2}$. For every $w \in L\left(\mathcal{A}^{\prime}\right)$, we have $\delta\left(q_{I}, w\right) \in F$, and thus, $w \in L$. 
Let $w=a_{1} \ldots a_{|w|} \in L$. There is a successful path in $\mathcal{A}^{\prime}$ with the label $w$ from $q_{I}^{\prime}$ along the states $\left(\left\{\delta\left(q_{I}, a_{1} \ldots a_{i}\right)\right\},\left\{\delta\left(q_{I}, a_{1} \ldots a_{i}\right)\right\}\right)$ for $1 \leq i \leq|w|$. Hence, $w \in L\left(\mathcal{A}^{\prime}\right)$. To sum up, $L\left(\mathcal{A}^{\prime}\right)=L$.

Proposition 6. Let $d, n \geq 1$ and $w \in S_{d}\left(\varrho_{n}(\mathcal{A})\right)$. There is a successful path $\pi$ in $\mathcal{A}^{\prime}$ with the label $w$ and $\Delta(\pi)<d+n$. In particular, $\pi$ contains at most $n$ péages and at most $d-1$ consecutive non-water transitions.

Proof (sketch). Let $1 \leq k \leq n$ and $P_{0}, \ldots, P_{k} \in \mathcal{P}_{n e}(Q), P_{0}=\left\{q_{I}\right\}, P_{k} \subseteq F$ such that $w \in S_{d}\left(P_{0}, \ldots, P_{k}\right)$. We can assume that for $2 \leq i \leq k, P_{i-1} \neq P_{i}$. For $1 \leq i \leq k$, let $w_{i} \in S_{d}\left(P_{i-1}, P_{i}\right)$ such that $w=w_{1} \ldots w_{k}$.

Let $2 \leq i \leq k$. There are an $a_{i} \in \Sigma$, some $k_{i} \geq 0$, and $w_{i, 1}, \ldots, w_{i, k_{i}}$ such that $w_{i}=a_{i} w_{i, 1} \ldots w_{i, k_{i}}, \delta\left(P_{i-1}, a_{i}\right) \subseteq P_{i}$, and for every $1 \leq j \leq k_{i}, \delta\left(P_{i}, w_{i, j}\right) \subseteq P_{i}$ and $\left|w_{i, j}\right| \leq d$. There is a transition $\left(\left(P_{i-1}, P_{i-1}\right), a_{i},\left(P_{i}, P_{i}\right)\right)$ in $\mathcal{A}^{\prime}$. It is both a water transition and a péage. For every $1 \leq j \leq k_{i}$, there is a path $\pi_{i, j}$ in $\mathcal{A}^{\prime}$ from $\left(P_{i}, P_{i}\right)$ to $\left(P_{i}, P_{i}\right)$ with the label $w_{i, j}$. These paths $\pi_{i, j}$ visit only states of the form $\left(R, P_{i}\right)$ for some $R \in \mathcal{P}_{n e}(Q)$. Hence, these paths do not contain any péage, and the last transition of each $\pi_{i, j}$ is a water transition. It is possible that $\left(P_{i}, P_{i}\right)$ occurs inside the paths $\pi_{i, j}$. Hence, it is possible that some path $\pi_{i, j}$ contains more than one water transition. Let $\pi_{i}=\left(\left(P_{i-1}, P_{i-1}\right), a_{i},\left(P_{i}, P_{i}\right)\right) \pi_{i, 1} \ldots \pi_{i, k_{i}}$. The label of $\pi_{i}$ is $a_{i} w_{i, 1} \ldots w_{i, k_{i}}=w_{i}$.

Similarly, we construct a path $\pi_{1}$ which starts in $q_{I}^{\prime}$, ends in $\left(P_{1}, P_{1}\right)$, and is labeled with $w_{1}$. We prove the proposition by setting $\pi=\pi_{1} \ldots \pi_{k}$.

Proposition 7. Let $w \in L\left(\mathcal{A}^{\prime}\right)$. We have $w \in S_{\Delta(w)}\left(\varrho_{\Delta(w)}(\mathcal{A})\right)$.

Proof (sketch). Let $\pi$ be a successful path in $\mathcal{A}^{\prime}$ with the label $w$ and $\Delta(\pi)=$ $\Delta(w)$. Let $k \leq \Delta(w)$ be the number of péages in $\pi$. We split $\pi$ into $\pi=\pi_{1} \ldots \pi_{k}$ such that every path $\pi_{1}, \ldots, \pi_{k}$ starts with a péage.

Let $P_{0}=\left\{q_{I}\right\}$. For every $1 \leq i \leq k$, the path $\pi_{i}$ ends in a state of the form $\left(P_{i}, P_{i}\right)$ for some $P_{i} \in \mathcal{P}_{n e}(Q), \pi_{1}, \ldots, \pi_{k}$ start with a péage and $\pi_{k}$ ends in an accepting state. We have $P_{k} \subseteq F$. For every $1 \leq i \leq k$, one can show that the label of $\pi_{i}$ belongs to $S_{\Delta(w)}\left(P_{i-1}, P_{i}\right)$. Thus, $w \in S_{\Delta(w)}\left(P_{0}, \ldots, P_{k}\right)$. Because $P_{0}, \ldots, P_{k}$ belongs to $\varrho_{\Delta(w)}(\mathcal{A})$, we have $w \in S_{\Delta(w)}\left(\varrho_{\Delta(w)}(\mathcal{A})\right)$.

By $L=L\left(\mathcal{A}^{\prime}\right)$ and Prop. 5 , 7 we obtain the following corollary:

Corollary 2. We have $\operatorname{sh}(L) \leq 1$ iff $\mathcal{A}^{\prime}$ is limited.

Proof (Theorem Q ). If $L$ is finite, then $\operatorname{sh}(L)=0$. If $L$ is infinite, then we construct $\mathcal{A}^{\prime}$. By Corollary 2, it suffices to decide whether $\mathcal{A}^{\prime}$ is limited. A total deterministic automaton for $L$ has at most $2^{n}$ states. Hence, $\mathcal{A}^{\prime}$ has at most $4^{2^{n}}+1$ state. By Theorem 1 we can decide in time complexity $2^{2^{2^{\mathcal{O}(n)}}}$ whether $\mathcal{A}^{\prime}$ is limited. 


\section{Next Research Steps and Open Problems}

In a forthcoming paper [7], we generalize the ideas of the present paper to achieve a new algorithm for the star height $n$ problem.

It is an open question whether the limitedness problem for distance desert automata is in PSPACE. We do not even know whether the limitedness problem for desert automata is in PSPACE. Another open question is whether there is an algorithm for the star height 1 problem with a better complexity.

Acknowledgments. The author acknowledges the discussions with JEAN-ERIC PIN and the remarks of anonymous referees on the paper. The author thanks IGOR WALUKIEWICZ for the permission to use one additional page.

\section{References}

[1] F. Dejean and M. Schützenberger. On a question of Eggan. Information and Control, 9:23-25, 1966.

[2] L. C. Eggan. Transition graphs and the star height of regular events. Michigan Math. Journal, 10:385-397, 1963.

[3] K. Hashiguchi. Limitedness theorem on finite automata with distance functions. Journal of Computer and System Sciences, 24:233-244, 1982.

[4] K. Hashiguchi. Regular languages of star height one. Information and Control, 53:199-210, 1982.

[5] D. Kirsten. Desert automata I. A Burnside problem and its solution. LIAFA Tech. Rep. 2003-020, Paris, 2003, (extended abstract appeared in V. Diekert and M. Habib, eds., STACS'04 Proceedings, LNCS, Springer-Verlag, Berlin, 2004).

[6] D. Kirsten. Desert automata II. A Burnside approach to the star height one problem. LIAFA Technical Report 2003-021, Paris, 2003.

[7] D. Kirsten. Desert automata III. Towards a comprehension of the star height problem. Manuscript, 2004.

[8] D. Krob. The equality problem for rational series with multiplicities in the tropical semiring is undecidable. Int. J. of Algebra and Computation, 4(3):405-425, 1994.

[9] H. Leung. An Algebraic Method for Solving Decision Problems in Finite Automata Theory. PhD thesis, Pennsylvania State Univ., Dep. of Computer Science, 1987.

[10] H. Leung. The topological approach to the limitedness problem on distance automata. In J. Gunawardena, ed., Idempotency, p. 88-111. Cambridge Univ., 1998.

[11] H. Leung and V. Podolskiy. The limitedness problem on distance automata: Hashiguchi's method revisited. Theor. Comp. Science, 310(1-3):147-158, 2004.

[12] S. Lombardy. Approche structurelle de quelques problèmes de la théorie des automates. PhD thesis, École nationale supérieure des télécomm., Paris, 2001.

[13] J.-E. Pin. Varieties of Formal Languages. North Oxford Academic Publ., 1986.

[14] I. Simon. Limited subsets of a free monoid. In Proceedings of the 19th IEEE Annual Symposium on Foundations of Computer Science, p. 143-150. 1978.

[15] I. Simon. Recognizable sets with multiplicities in the tropical semiring. In M. P. Chytil et al., ed., MFCS'88 Proc., LNCS 384, p. 107-120. Springer-Verlag, 1988.

[16] I. Simon. On semigroups of matrices over the tropical semiring. Informatique Théorique et Applications, 28:277-294, 1994. 